\title{
Do cochlear implants play a role in the induction of brain tumors?
}

\author{
Thomas Mindermann ${ }^{1}$
}

Received: 20 January 2016 / Accepted: 22 January 2016 / Published online: 3 February 2016

(C) Springer-Verlag Wien 2016

\section{Editorial}

In this issue of Acta Neurochirurgica, Kalkoti et al. [2] report two cases of glioblastoma multiforme (GBM) diagnosed in patients with cochlear implants (CIs). These are the first two cases in the literature making a connection between $\mathrm{CI}$ and the development of brain tumors.

As the authors report in their paper, until today more than 500,000 patients have received CI worldwide. At the same time, the overall incidence of primary malignant brain tumors is about 7 per 100,000 person-years, and the prevalence is about 29 per 100 , 000 inhabitants in the USA $[1,3]$. The most common brain tumors are meningiomas in patients older than 20 years of age. The second most common brain tumors are nerve sheath tumors and glioblastomas in patients 35-44 years of age, and glioblastomas are the second most common brain tumors in all patients older than 45 years of age [1,3]. Glioblastomas are more common in male patients with a female-to-male ratio of $1: 1.6[1,3]$. Therefore, 1 GBM in 250,000 patients with $\mathrm{CI}$ is not necessarily an alarming finding from an epidemiological point of view.

However, if one takes the demographics of this publication into account, the two cases may deserve a closer look. One of the patients was a 60-year-old male and the other a 42-year-old female. While the 60-year-old

Thomas Mindermann tmindermann@hin.ch

1 Neurosurgery, Klinik Im Park, Seestrasse 220, 8027 Zurich, Switzerland male patient fits the expected demographic pattern of patients with glioblastomas, the 42-year-old female patient does not quite as well. For this and other reasons, the authors' observation should not be easily dismissed as anecdotical. So far, a number of adverse effects have been associated with $\mathrm{CI}$ but not the induction of brain tumors. Therefore, it is likely that a possible connection between $\mathrm{CI}$ and the induction of brain tumors has been underreported so far because no one ever thought about the possibility of a causal relationship. Another point that deserves attention is the latency period from the implant of the CI to the diagnosis of GBM in these two patients, which was 7 and more than 23 years, respectively. This latency period is suggestive of a causal relationship just like the GBMs' occurrence in close proximity to the CI transmitting devices on the skull bone. Furthermore, we know that the external application of alternating electrical fields (AEFs) to the skull interferes with the ability of deep-seated intraparenchymal tumor cells to replicate [4]. Obviously, this kind of treatment has an effect at the subcellular level, influencing the cells' mitotic processes. The question arises whether the application of continuous, long-term, external electrical fields to the brain as seen in CI may have a detrimental effect on the brain parenchyma, placing patients at higher risk for brain tumor induction.

In conclusion, because of the demographics, tumor location, latency period, and long-term application of electrical fields to the brain, as reported in these two patients with $\mathrm{CI}$, the authors may have found a problem deserving our attention. A heightened vigilance of the neurosurgical community may be indicated since neurosurgeons are the most likely specialists to see patients with $\mathrm{CI}$ and brain tumors. 


\section{References}

1. CBTRUS (2004) Statistical report: primary brain tumors in the United States, 1997-2001. Published by the Central Brain Tumor Registry of the United States

2. Kalakoti P, Murray RD, Pettersson-Segerlind J, Smeds H, Nanda A (2016) Cochlear implants in the etio-pathogenesis of glioblastomaan interesting observation or an independent finding? Acta Neurochir (in press)
3. Ostrom QT, Gittleman H, Fulop J, Liu M, Blanda R, Kromer C, Wolinsky Y, Kruchko C, Barnholtz-Sloan JS (2015) CBTRUS statistical report: primary brain and central nervous system tumors diagnosed in the United States in 2008-2012. Neuro-Oncology 17: iv1-iv62. doi:10.1093/neuonc/nov189

4. Rehman AA, Elmore KB, Mattei TA (2015) The effect of alternating electric fields in glioblastoma: current evidence on therapeutic mechanisms and clinical outcomes. Neurosurg Focus 38:E14-E24 